\section{Desensitization to Hydroxychloroquine: 4 Cases}

\section{To the Editor:}

Successful desensitization to hydroxychloroquine (HCQ) was reported in The Journal in $2006^{1}$; the authors described 2 patients with systemic lupus erythematosus (SLE) and 2 patients with polyarthritis who were given HCQ. The drug was withdrawn 7-14 days later due to the appearance of a maculopapular rash. After resolution of the skin lesions all 4 patients again received HCQ at an initial dose of $0.1 \mathrm{mg} / \mathrm{day}$; the dosage was progressively increased to $400 \mathrm{mg} /$ day; only one patient showed a transitory rash, which did not require interruption of treatment.

Recently, another case appeared of a patient with SLE who successfully underwent HCQ desensitization ${ }^{2}$; the patient developed anaphylaxis after resumption of the drug following a 2-week interruption of treatment. Before the procedure the patient showed a positive skin-prick test to HCQ; the test became negative after the end of the desensitization process.

We applied the same protocol suggested by Mates, et $a l^{1}$ to 4 patients who developed a maculopapular rash 10-15 days after the initiation of HCQ treatment. All patients were young females; one had SLE, 2 a lupus-like syndrome, and one primary Sjögren's syndrome. All stopped HCQ treatment after the appearance of the cutaneous manifestations. In all the patients, HCQ therapy was later restarted at the dose of $0.1 \mathrm{mg}$ on the first day, $0.2 \mathrm{mg}$ the second day, and $0.4 \mathrm{mg}$ the third day; the dosage was progressively increased to $200 \mathrm{mg} /$ day by Day 32 . No premedication with antihistamine or other drug was given. In no case did the rash relapse.

During the first 3 days the patients were monitored in our unit for 3 hours after administration of the drug with a peripheral intravenous line. The desensitization process was then completed by the patients at home.

For the procedure, tablets of $0.1 \mathrm{mg}$ and $2 \mathrm{mg}$ were prepared by a hospital chemist, simply by cutting tablets available on the market.

We agree with Sontheimer's comments ${ }^{3}$ regarding the report from Mates and coworkers, that viral infections may simulate hypersensitivity drug reactions and that drug-induced rashes rarely reappear following readministration of the offending drug. One cannot ascribe the occurrence of a rash that appears some days after the initiation of HCQ to the drug with absolute certainty, and the adverse reaction may not always reappear after rechallenge. Nevertheless, from a clinical viewpoint this should not dimin- ish the relevance of the desensitization process, considering that in a few cases the rechallenge may be followed by severe life-threatening manifestations such as exfoliative erythroderma.

HCQ is used extensively in rheumatology, with a favorable benefit/risk ratio; it is well known that the risk of ocular toxicity is lower than that caused by chloroquine. The unique characteristics of the drug make it irreplaceable; the majority of patients with SLE receive HCQ alone or in combination with other therapies.

The possibility of performing a desensitization procedure allows the drug to be restarted in a more safe and gradual manner, providing the patient an opportunity to continue this useful therapy. Our experience confirms the validity of this procedure, which was managed on an outpatient basis, not involving hospitalization. A similar approach may be performed in cases of skin reactions after allopurinol administration ${ }^{4}$.

PAOLA CARAMASCHI, MD, Rheumatology Unit; ROBERTO BARBAZZA, MD, Pharmacy Service; ILARIA TINAZZI, MD; DOMENICO BIASI, MD, Rheumatology Unit, Medicine Department, University of Verona, Verona, Italy. Address correspondence to Dr. P. Caramaschi, Unità di Reumatologia, Policlinico G.B. Rossi, Piazzale Scuro, 37134 Verona, Italy.

E-mail: paola.caramaschi@ospedaleuniverona.it

\section{REFERENCES}

1. Mates M, Zevin S, Breuer GS, Navon P, Nesher G. Desensitization to hydroxychloroquine - experience of 4 patients. J Rheumatol 2006;33:814-6.

2. Sontheimer RD. Desensitization to hydroxychloroquine: alternative interpretations [editorial]. J Rheumatol 2007;34:253-5.

3. Díaz Donado C, Maquiera Díez E. Successful desensitization for hydroxychloroquine anaphylaxis. J Rheumatol 2010;37:1975-7.

4. Fam AG, Dunne SM, Iazzetta J, Paton TW. Efficacy and safety of desensitization to allopurinol following cutaneous reactions. Arthritis Rheum 2001;44:231-8.

J Rheumatol 2011;38:10; doi:10.3899/jrheum.110345 